\title{
Small Peptides for Inhibiting Serum Amyloid A Aggregation
}

\author{
Asis K. Jana, Augustus B. Greenwood and Ulrich H. E. Hansmann* \\ Department of Chemistry \& Biochemistry, University of Oklahoma, Norman, OK 73019, USA. \\ KEYWORDS: Molecular Dynamics Simulations, Serum Amyloid A
}

\begin{abstract}
Deposition of human Serum Amyloid A (SAA) amyloids in blood vessels, causing inflammation, thrombosis and eventually organ damage, are a commonly seen as a consequence of certain cancers and inflammatory diseases. Several attempts have been made to develop peptide-based drugs that inhibit or at least slow down SAA amyloidosis. We use extensive all-atom molecular dynamic simulations to compare three of these drug candidates for their ability to destabilize SAA fibrils, and to propose for the best candidate, the $\mathrm{N}$-terminal sequence $\mathrm{SAA}_{1-5}$, a mechanism for inhibition. As the life-time of peptide drugs can be increased by replacing L-amino acids with their mirror Damino acids, we have also studied corresponding D-peptides. We find that DRI-SAA $1-5$, formed of D-amino acids with the sequence of the peptide reversed, has similar inhibitory properties than the original L-peptide, and therefore may be a promising candidate for drugs targeting SAA amyloidosis.
\end{abstract}




\section{Introduction}

Various cancer and inflammatory diseases can promote overexpression of serum amyloid $\mathrm{A}^{1,2}$ to 1000 times higher concentrations than seen usually ${ }^{3}$. The resulting higher chance for misfolding and aggregation leads often to SAA amyloidosis as a secondary disease bringing on wide-spread damage to tissues and organs, with kidney failure a common complication. ${ }^{4}$ Similar SAA concentrations have been also reported after SARS-COV-2 infections ${ }^{5,6,7}$, suggesting an increased risk for SAA amyloidosis ${ }^{8}$. Hence, there is an interest in finding drug candidates that can inhibit or at least slow down SAA amyloid formation.

Several attempts have been made to develop such drugs based on synthesis of small peptides. These peptides are chosen from the sequence of amyloidogenic proteins for their complementarity to the regions on the SAA protein that are responsible for self-association ${ }^{9-13}$. This peptide-based approach has been successfully applied to combat aggregation of amyloid- $\beta$ (A $\beta$ ) peptides (connected to Alzheimer's disease) and $\alpha$-synuclein (implied in Parkinson's disease), where sodesigned inhibitors are able to prevent fibril formation and to disaggregate preformed fibrils, both in cell culture models and in animal models ${ }^{9-15}$. Earlier biophysical studies have confirmed that the N-terminal sequence RSFFS of the SAA protein and the $A \beta$ peptide segment $A \beta_{17-20}$ (LVFF) can significantly suppress the SAA aggregation process ${ }^{10}$. However, that study considered as drug target only the short $\mathrm{SAA}_{1-12}$ fragment ${ }^{16,17}$, which has different aggregation propensity than larger segments. ${ }^{12}$ For this reason, we choose in the present study assemblies of larger SAA fragments as drug targets, but select again these two peptides as possible drug candidates. In addition, we add as a third inhibitor candidate the peptide FVFLM of the protein SERPINA1, found in in urine and placenta ${ }^{18}$ of pregnant women suffering from preeclampsia. Our choice is motivated by an in silico study of Kouza et. al. ${ }^{19}$ who showed that FVFLM strongly binds with central hydrophobic 
domain $A \beta_{16-20}$ (KLVFF), thereby disrupting the $A \beta$ aggregation pathway. We hypnotize that a similar mechanism is likely for inhibition of SAA aggregation. All three inhibitor candidates are listed in Table 1 together with their names used in this study.

Table 1: Inhibitor candidates evaluated in this study

\begin{tabular}{|c|c|c|}
\hline Name & Sequence & Source \\
\hline R5S & RSFFS & SAA $_{1-5}$ \\
\hline L4F & LVFF & $\mathrm{A} \beta_{17-20}$ \\
\hline F5M & FVFLM & SERPINA protein \\
\hline
\end{tabular}

SAA fibrils are not formed by the full-sized protein, but by shorter fragments derived after enzymatic cleavage. The most common fragment is $\mathrm{SAA}_{1-76}$, but the only experimentally resolved human SAA fibril structure is for the segment $\mathrm{SAA}_{2-55}$, deposited in the Protein Data Bank under identifier $6 \mathrm{MST}^{20}$. Hence, in the present paper we compare through molecular dynamics simulations the ability of the three inhibitor candidates to reduce the stability of this fibril structure, a necessary condition for lowering the risk for amyloidosis.

Our results suggest that the fragment $\mathrm{SAA}_{1-5}$ (RSFFS, to which we refer in the following as R5S) has a larger effect of fibril stability than L4F (the LVFF segment $A \beta_{17-20}$ ) and F5M, the segment FVFLM of SEREPINA1. Since short half-life time and the resulting need for frequent drug administration are common complications in peptide-based therapies that would also hamper the use of R5S as a potential drug, we have also studied versions of R5S where the L-amino acids are replaced by D-amino acids. This is because peptides made of D-amino acids are resistant to 
proteolytic digestion, resulting in longer half-life times. Hence, following our earlier work ${ }^{21}$ we have studied both a variant where the sequence is unchanged (D-R5S), and one where also the sequence is reversed (DRI-R5S). The later variant is chosen because such D-retro-inverso peptides resemble the parent peptide and have often similar biological activity ${ }^{22-24}$. We find that DRI-R5DS is similar effective in destabilizing SAA fibrils than the original L-peptide, and therefore may be a promising candidate for drugs targeting SAA amyloidosis.

\section{Materials and Methods}

\section{System Preparation}

We have evaluated the ability of three peptides to inhibit Serum Amyloid A (SAA) amyloid formation. Three candidates were selected after screening recent publications ${ }^{10}$ and are listed in Table 1. A posteriori, two additional peptides were considered where we replaced the L-amino acids of the best performing candidate by D-amino acids, either in the same or with reverse sequence. Such D or DRI peptides are difficult to proteolyze and therefore have longer life times in the cell.

Assuming that potential inhibitors destabilize the fibril architecture, we have compared all-atom molecular dynamics simulations of SAA fibril fragments in the presence of one of these candidate inhibitors with such where the inhibitor candidates are absent. The fibril fragment is derived from the cryo-EM structure as deposited in the Protein Data Bank (PDB) under identifier 6 $\mathrm{MST}^{20}$, presently the only available experimentally derived human SAA fibril model. While this model describes a fibril made of $\mathrm{SAA}_{2-55}$ fragments instead of the more commonly found $\mathrm{SAA}_{1-76}$ fragments, we did not attempt to add additional residues by homology modeling. This is because 
we had found in earlier work ${ }^{25}$ that the disordered C-terminal tail of residues 56-76 does not alter by itself the stability of the fibril. Similarly guided by our previous work ${ }^{25}$ we choose a two-foldthree-layer $(2 \mathrm{~F} 3 \mathrm{~L})$ hexamer as model for our simulations. This is because this hexamer is above the critical threshold for a stable SAA fibril fragment (which we found in our earlier work to be the two-fold-two-layer (2F2L) tetramer). Note that in some exploratory simulations we also considered the hexamer with the N-terminal Arginine add to all six chains by Chimera ${ }^{26}$, and the resulting configuration minimized under the constraint that the experimentally resolved parts of the structure stay unchanged.

We then used Autodock Vina ${ }^{27}$ to identify initial binding sites on the fibril for the inhibitors, before preparing start configurations for our simulations where the respective inhibitor candidates are bound to the SAA chains in the fibril at a 1:1 ratio. In the so-obtained configuration are the SAA chains capped by $\mathrm{NH}_{3}{ }^{+}$and $\mathrm{COO}^{-}$, while the $\mathrm{N}$ - and C- termini of inhibitors are capped by $\mathrm{NH}_{2}$ and $\mathrm{CONH}_{2}$ to counteract the strong electrostatic repulsion between the terminal groups. The resulting systems are then put into a box with periodic boundary conditions and with a box size, large enough that there is at least $15 \AA$ distance between any atom on the system and a box side. The box is filled with TIP3 $\mathrm{P}^{28}$ water molecules and a suitable number of $\mathrm{Na}+$ and $\mathrm{Cl}$ - ions to obtain the desired ion concentration, in our case either $0 \mathrm{M}$ or $150 \mathrm{mM}$. We list all systems, together with the number of water molecules, total number of atoms, and ion concentration in Table S1.

\section{Simulation Protocol}

For our simulations we use the GROMACS 2018 simulation package, while configurations were visualized using Visual Molecular Dynamics (VMD). SAA fibrils and peptide inhibitors are parametrized with the CHARMM 36m all-atom force-field, which is consistent with the TIP3P 
water model used by us. Initially, each system is energy minimized by the steepest-descent algorithm to remove bad contacts between the solute and solvent, before equilibrated over $200 \mathrm{ps}$ in a (NVT) ensemble at a constant temperature of $310 \mathrm{~K}$, and additional 200ps in an isothermalisobaric (NPT) ensemble at a constant pressure of $1 \mathrm{~atm}$. The nonhydrogen (heavy) atoms of fibril and inhibitors are held fixed during equilibration with a force constant of $1,000 \mathrm{~kJ} \mathrm{~mol}^{-1} \mathrm{~nm}^{-2}$. The resulting configurations are the start point for the subsequent production runs performed at $310 \mathrm{~K}$ and $1 \mathrm{~atm}$, with the temperature and pressure controlled by the v-rescale thermostat ${ }^{29}$ (with a coupling constant of $0.1 \mathrm{ps}$ ) and the Parrinello-Rahman barostat ${ }^{30}$ (with a pressure relaxation time of $2 \mathrm{ps).} \mathrm{Non-water} \mathrm{bonds} \mathrm{involving} \mathrm{hydrogen} \mathrm{atoms} \mathrm{are} \mathrm{constrained} \mathrm{using} \mathrm{the} \mathrm{LINCS} \mathrm{algorithm}{ }^{31}$, and water molecules kept rigid by the SETTLE algorithm, ${ }^{32}$ allowing an integration time step of 2 fs. As we use periodic boundary conditions, we employ the particle-mesh Ewald (PME) method ${ }^{33}$ for calculating long-range electrostatic interactions. The cutoff for Van der Waal interactions is set to $12 \AA$, with the smoothing startin at $10.5 \AA$.

\section{Trajectory analysis}

For most of our analysis we use GROMACS tools such as gmx_rms which calculates the rootmean-square deviation (RMSD) with respect to an initial configuration. For visualization we use the VMD software, which we use also to calculate the solvent-accessible surface area (SASA), selecting a spherical probe of $1.4 \AA$ radius. ${ }^{34}$ We have estimated interpeptide binding free energy $(\Delta G)$ between SAA chains using the Molecular Mechanics Poisson-Boltzmann surface area (MMPBSA) $)^{35}$ approach as implemented in the GROMACS package. ${ }^{36}$ In this method, $\Delta G$ is approximated by:

$$
\Delta G=\Delta H_{M M}+\Delta G_{\text {solv-pol }}+\Delta G_{\text {solv-np }}
$$




$$
\Delta H_{M M}=\Delta H_{\text {elec }}+\Delta H_{v d w},
$$

Here, $\Delta \mathrm{H}_{\mathrm{MM}}$ represents the potential energy difference between fibril and the free chains in vacuum, which is the sum of electrostatic $\left(\Delta \mathrm{H}_{\text {elec }}\right)$ and van der Waals energy $\left(\Delta \mathrm{H}_{\mathrm{vdw}}\right)$ differences. $\Delta \mathrm{G}_{\text {solv-pol }}$ represents the change in polar solvation free energy and is estimated by solving the nonlinear Poisson-Boltzmann equation at the solvent dielectric constant of water at $310 \mathrm{~K}$, i.e., $\varepsilon$ $=78 . \Delta \mathrm{G}_{\text {solv-np }}$ represents the change in non-polar solvation free energy and is assumed to be proportional to the solvent accessible surface area (SASA). $\Delta G$ is computed over the three independent trajectories for each system, considering only the final $100 \mathrm{~ns}$.

\section{Results}

\section{Optimizing the stability of the benchmark fibril system}

Amyloid formation decreases the entropy of the SAA chains, and overcoming this loss of entropy therefore requires an energetic bias toward the fibril structure. Peptide inhibitors work by reducing the energetic stabilization of the fibril structure upon binding to the fibril. Hence, for evaluating the peptide inhibitor candidates we have to ensure first that in our simulations the fibril stability is not already artificially decreased by other interfering factors such as, for instance, the chosen ionic strength. Another potential systematic error could result from the missing first residue in the experimentally determined $\mathrm{SAA}_{2-55}$ fibril model. This residue, an Arginine, is at neutral $\mathrm{pH}$ protonated, and therefore able to form salt bridges with negatively charged residues. These salt bridges are missing in simulations of our model which as a consequence may underestimate the stability of the fibril. In order to exclude such systematic errors we first compare simulations of the fibril with and without the N-terminal Arginine; and without added salt (except for counterions) and at $150 \mathrm{mM} \mathrm{NaCL}$ (the physiological intra-cellular salt concentration). The time evolution of 
the root-mean-square deviation (RMSD) to the respective start configuration is shown in Figure 1. As the RMSD is calculated over all the backbone atoms and all chains in the respective fibril model, we call it a global RMSD.

Comparing the four simulations we observe first that the RMSD is lower in simulations of the fibril with physiological salt concentration than it is in the simulations of the corresponding systems without salt. Hence, omitting salt artificially decreases fibril stability. While this effect is small in simulations where the first residue is missing, raising from $(4.4 \pm 0.4) \AA$ in $0 \mathrm{mM}$ to (4.7 \pm 0.4$) \AA$ in $150 \mathrm{mM}$, it is more pronounced in simulations where the Arginine is added. Here, the final RMSD value at $100 \mathrm{~ns}$ is $(5.8 \pm 0.4) \AA$ in $0 \mathrm{mM} \mathrm{NaCl}$ and $(5.0 \pm 0.8) \AA$ in $150 \mathrm{mM} \mathrm{NaCl}$.

Similarly, we find in accordance with previous experimental results ${ }^{20}$ that addition of the Nterminal arginine reduces the stability of the fibril even when comparing the two models at the same salt concentrations. Presence of arginine breaks stabilizing contacts within the segment of residues 5-15, see Figure S1 in the supporting information, with the effect lower when the electrostatic interactions are screened by the $\mathrm{Na}^{+}$and $\mathrm{Cl}^{-}$ions. We remark that by repeating the above four simulations now adding the R5S inhibitor, we find the same trend as for the control simulations (in absence of any inhibitor), see Supplemental Figure S2. Hence, for our evaluation and the comparison of the inhibitors, relying on longer trajectories, we use the experimental fibril model without added N-terminal arginine, and choose a salt concentration of $150 \mathrm{mM}$. 
(a)

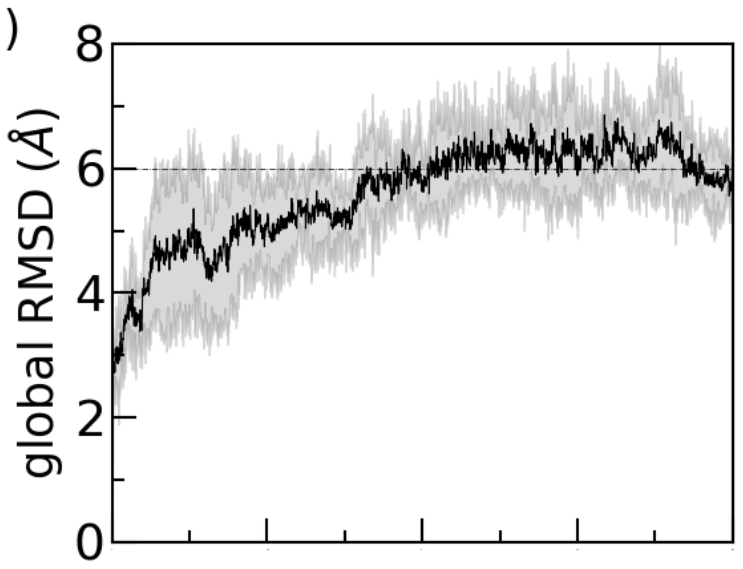

(c)

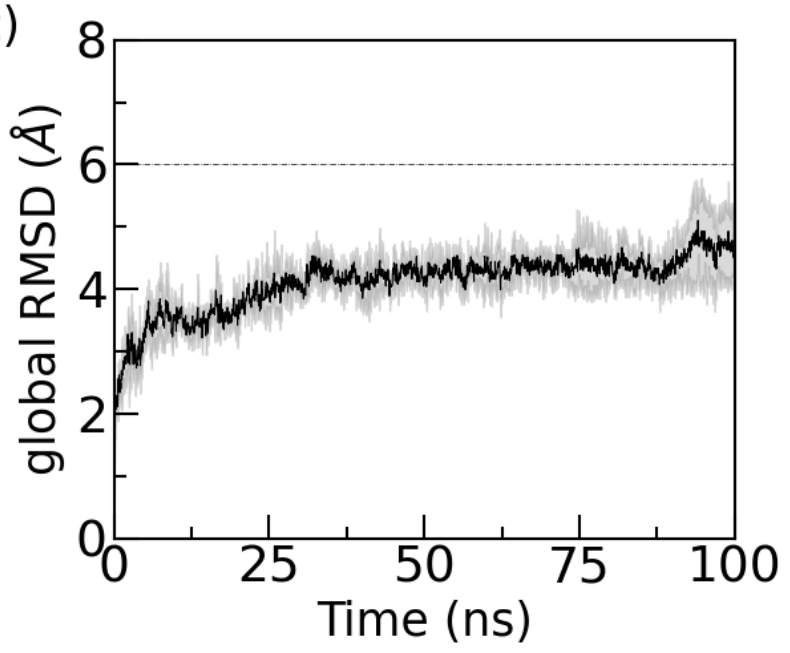

(b)

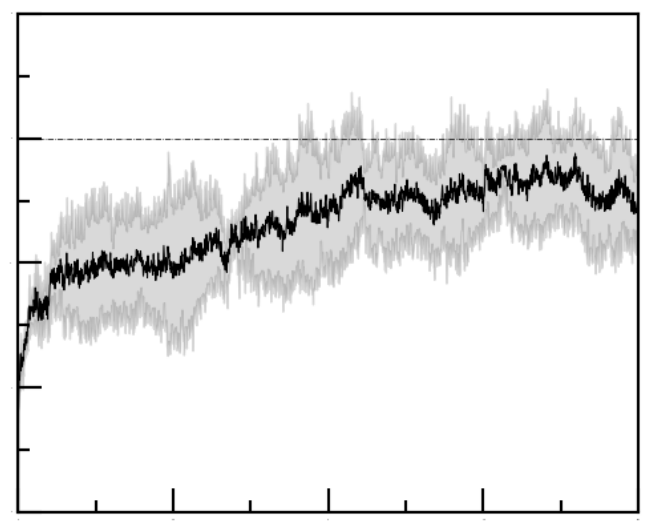

(d)

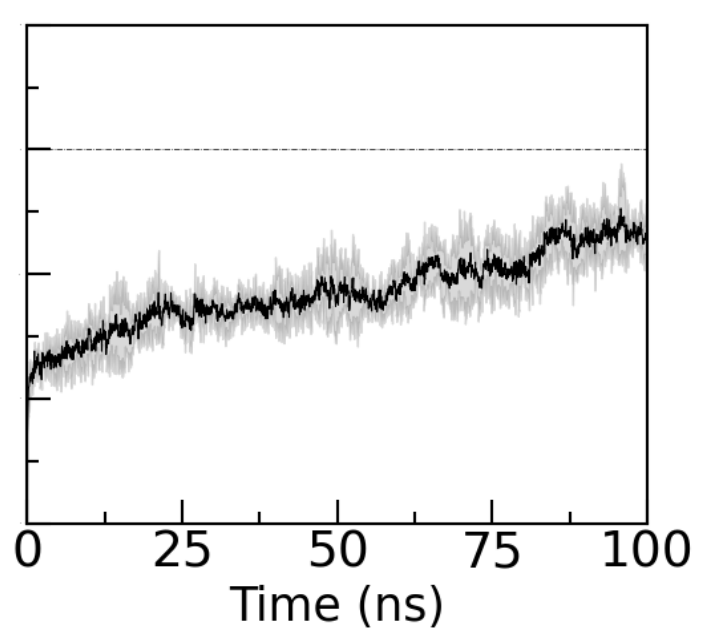

Figure 1: Time evolution of global RMSD of control simulations. RMSD values are calculated in reference to the experimentally resolved structure (PDB ID: 6MST) considering only backbone atoms in all chains of the respective fibril model. The data are averaged over three trajectories, with the shaded region indicating the standard deviation of the average. Systems displayed are those with arginine at $0 \mathrm{mM}$ (a), with arginine at $150 \mathrm{mM} \mathrm{NaCl}$ (b), without arginine at $0 \mathrm{mM}$ (c) and without arginine at $150 \mathrm{mM}(\mathrm{d})$. In order to allow for a more easy comparison of the four systems, we have drawn dashed lines at $6 \AA$ to guide the eyes.

\section{Comparing the effect of the three inhibitors on SAA fibril stability}

With the optimal simulation conditions determined, we then compare for all three inhibitor candidates the stability of the SAA fibril in presence of the inhibitor with the stability of the control. Our results are shown in Figure 2, where we show in the top row the global RMSD to the 
start configuration, and in the middle row the chain RMSD with respect to the start configuration. As in Figure 1 is the global RMSD calculated for the backbone atoms in all chains of the fibril model, whereas the chain RMSD is the average over chain-wise calculated RMSD values. Hence, the global RMSD measures the structural deviation of the entire fibril while the chain RMSD measures the structural distortion of each protein chain within the fibril. On the other hand, the residue-wise root-mean-square-fluctuation (RMSF) shown in the bottom row describes the flexibility of a certain residue in the chains that make up the fibril.

Focusing first on the top two rows in Figure 2 we see that global and chain RMSD are correlated. Both RMSD values are growing for all three inhibitors faster and to higher values than for the control, with the effect most pronounced for Inh1. For instance, the global RMSD values averaged over the final $100 \mathrm{~ns}$ and the three trajectories are for the control $(0.49 \pm 0.02) \mathrm{nm}$, for R5S $(0.74 \pm 0.05) \mathrm{nm}$, for $\mathrm{L} 4 \mathrm{~F}(0.66 \pm 0.03) \mathrm{nm}$ and for F5M $(0.60 \pm 0.04) \mathrm{nm}$. The correlation between global and chain RMSD is especially obvious for R5S inhibitor where the sudden rise in global RMSD at $200 \mathrm{~ns}$ is mirrored by one in chain RMSD. The correlation between the two quantities suggests that the dissolution of the fibril goes in hand with a change in chain structure. In order to quantify the structural change of the SAA chains we have also calculated the residue-wise backbone RMSF along the trajectories and shown this quantity in the bottom row of Figure 2. Here we find that the large structural deviations, seen by visual inspection in the $\beta$-arch formed by residues $2-22$, and in the C-terminal cavity formed by residues $45-55$, is correlated with high flexibility of the residues in these two regions. Note that the RMSF distribution of each residue is for each of the inhibitor simulations broader than for the control (the fibril without inhibitor candidate added). Hence, the inhibitor disturbs the chain conformations which in turn leads to dissolution of the fibril geometry. 

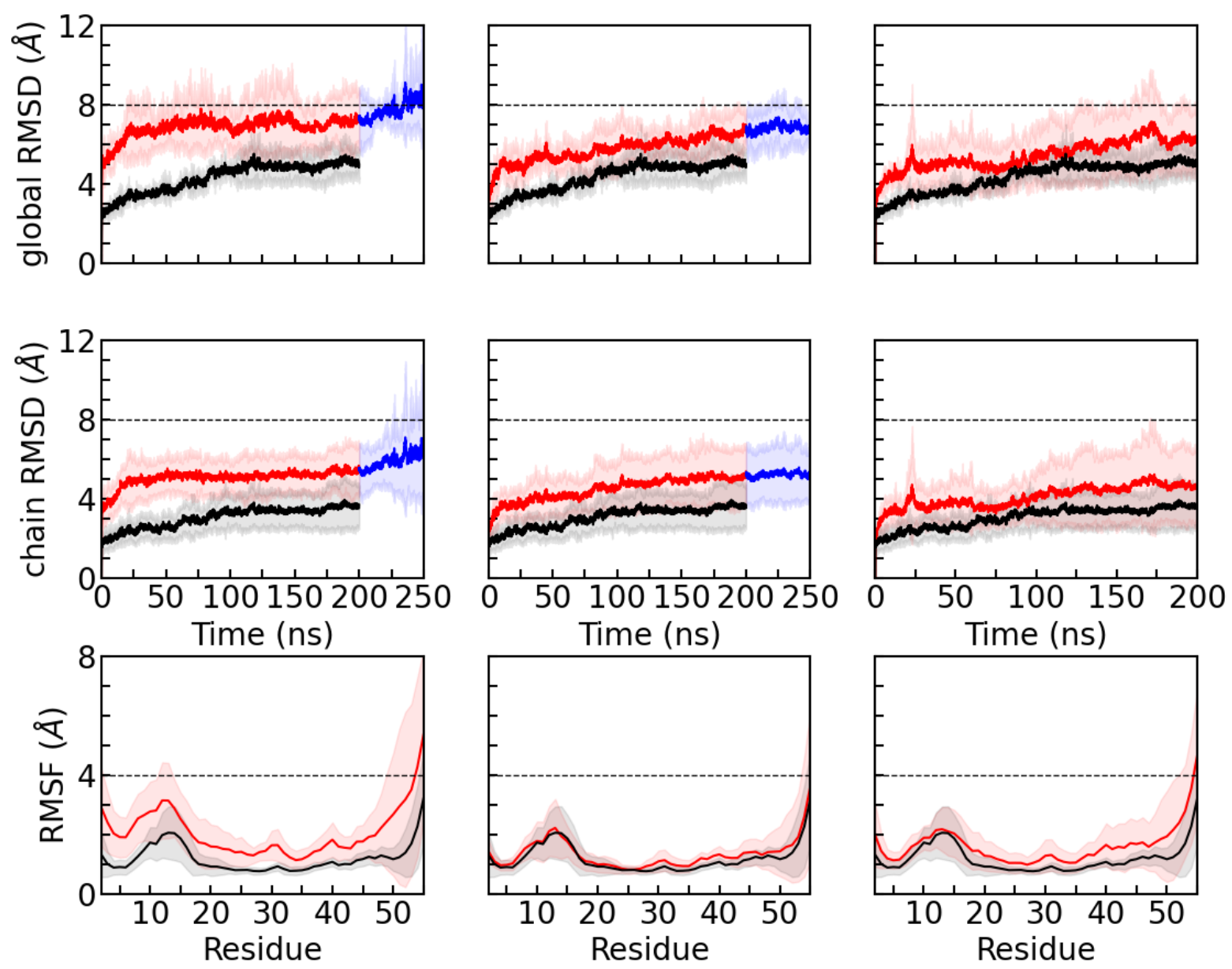

Figure 2: Time evolution of the global and chain RMSD measured in simulations of the fibril in absence (in black) and presence of three inhibitors (in red). Residue-wise RMSF values are calculated over the final $100 \mathrm{~ns}$ of each trajectory. Data in presence of R5S, L4F and F5M inhibitors are shown in the first, second and third column, respectively. The simulations with R5S and L4F inhibitors present were later extended up to $250 \mathrm{~ns}$. This additional timespan is drawn in blue. Chain RMSD and RMSF values are averaged over all six chains in the SAA fibril and all three trajectories. The shaded region represents the standard deviation. The RMSD and RMSF values are calculated in reference to the experimentally resolved structure considering all backbone atoms in residues 2-55. In order to allow for a more easy comparison of the four sys-tems, we have drawn dashed lines at suitable values of RMSD or RMSF to guide the eyes.

Note that we simulated initially the control and the inhibitor-containing for $200 \mathrm{~ns}$. As the differences between R5S and L4F inhibitors were small, and unlike the control or F5M inhibitor the RMSD in both systems appeared to continue growing, we extended simulations for R5S and L4F inhibitors by $50 \mathrm{~ns}$, with the extension drawn in a different color. With this extension, it 
appears from Figure 2 and visual inspection of the snapshots in Figure 3 that the structural deviations are largest in presence of R5S inhibitor.
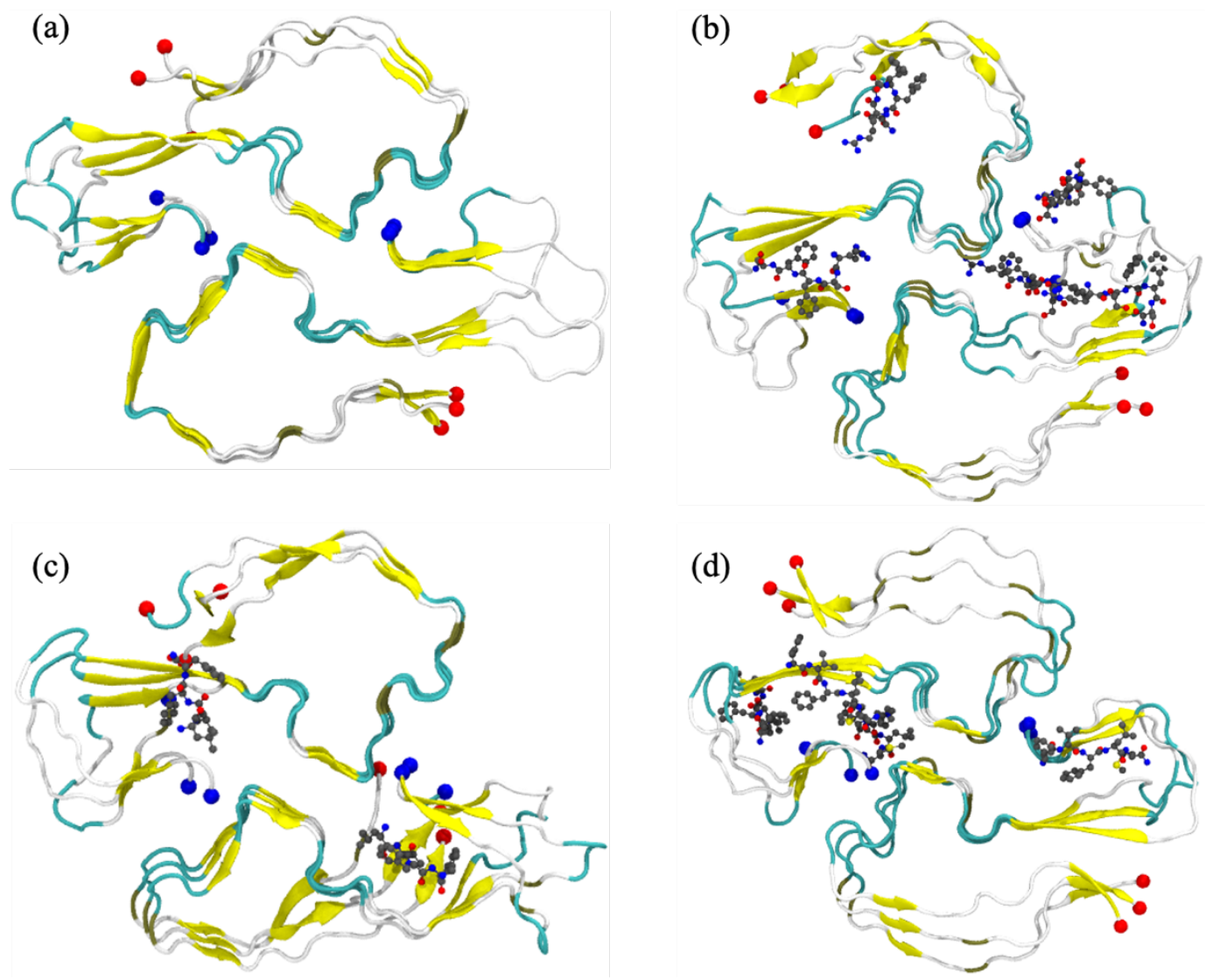

Figure 3: Representative final configuration extracted from a control simulation of the $\mathrm{SAA}_{2-55}$ fibril is shown in (a). Corresponding final snapshots extracted from simulations in presence of R5S, L4F and F5M inhibitors are shown in (b), (c) and (d), respectively. N- and C-terminal residues are marked by blue and red spheres, respectively.

We have quantified this observation by measuring for both the control and inhibitor simulations the solvent accessible surface area (SASA), number of stacking contacts $\left(\mathrm{N}_{\text {stacking }}\right)$, number of stacking hydrogen bonds ( $\left.\mathrm{N}_{\mathrm{HB} \text {-stacking }}\right)$, number of packing contacts $\left(\mathrm{N}_{\text {packing }}\right)$, number of packing hydrogen bonds ( $\mathrm{N}_{\text {HB-packing }}$, and the inter-strand packing distance ( $\left.\mathrm{D}_{\text {packing }}\right)$. We list in Table 2 
the mean and standard deviation of these quantities, as calculated over the last $100 \mathrm{~ns}$ of for each system three independent trajectories. The first of these quantities, the solvent-accessible surface area (SASA) of the hydrophobic residues, is a measure for the solvent exposure of the hydrophobic residues in the SAA fibril. It is measured in the VMD package by running a spherical probe of 1.4 $\AA$ radius over the fibrillar surface. In case of R5S inhibitor, the hydrophobic SASA increases by about $\sim 26 \%$ over the control, suggesting the inhibitor causes conformational changes in which the tightly packed hydrophobic core becomes hydrated. This is not the case for the other two inhibitors, where the SASA values increase by only about $10 \%$.

Table 2: Solvent accessible surface area (SASA), number of stacking contacts $\left(\mathrm{N}_{\text {stacking }}\right)$ and stacking hydrogen bonds ( $\left.\mathrm{N}_{\text {HB-stacking}}\right)$; number of packing contacts $\left(\mathrm{N}_{\text {packing }}\right)$ and packing hydrogen bonds ( $\mathrm{N}_{\text {HB-packing }}$ ); and of the inter-strand packing distance $\left(\mathrm{D}_{\text {packing }}\right)$. Averages are calculated over the three independent trajectories considering only the final $100 \mathrm{~ns}$ of each trajectory, with the corresponding standard deviations also shown. The change between simulations in presence and absence of the inhibitors is listed as percent changes.

\begin{tabular}{|c|c|c|c|c|}
\hline System & Control & R5S & L4F & F5M \\
\hline SASA $\left(\AA^{2}\right)$ & $6435 \pm 371$ & $8118 \pm 632$ & $7130 \pm 576$ & $7099 \pm 374$ \\
\hline$\% \Delta$ SASA & - & 26 & 11 & 10 \\
\hline $\mathrm{N}_{\text {stacking }}$ & $16100 \pm 376$ & $14389 \pm 612$ & $15562 \pm 414$ & $15118 \pm 861$ \\
\hline$\% \Delta \mathrm{N}_{\text {stacking }}$ & - & -11 & -3 & -6 \\
\hline $\mathrm{N}_{\text {packing }}$ & $2265 \pm 216$ & $1203 \pm 425$ & $1720 \pm 149$ & $1648 \pm 302$ \\
\hline$\% \Delta \mathrm{N}_{\text {packing }}$ & - & -47 & -24 & -27 \\
\hline $\mathrm{N}_{\text {HB-stacking }}$ & $56 \pm 7$ & $51 \pm 6$ & $53 \pm 7$ & $50 \pm 8$ \\
\hline$\% \Delta \mathrm{N}_{\text {HB-stacking }}$ & - & -9 & -5 & -10 \\
\hline $\mathrm{N}_{\text {HB-packing }}$ & $7 \pm 2$ & $5 \pm 2$ & $6 \pm 2$ & $5 \pm 2$ \\
\hline$\% \Delta \mathrm{N}_{\text {HB-packing }}$ & - & -30 & -18 & -34 \\
\hline $\mathrm{D}_{\text {packing }}(\AA)$ & $9.5 \pm 0.3$ & $12.2 \pm 4.0$ & $9.6 \pm 0.3$ & $9.7 \pm 0.4$ \\
\hline$\% \Delta \mathrm{D}_{\text {packing }}$ & - & 29 & 2 & 2 \\
\hline
\end{tabular}

VMD is also used to calculate the total number of stacking contacts $\left(\mathrm{N}_{\text {stacking }}\right)$, stacking hydrogen

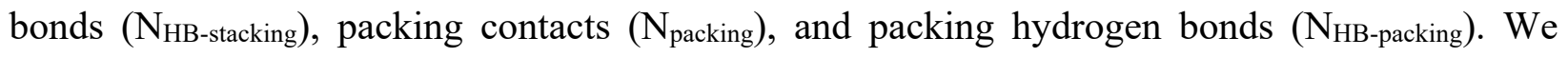
define contacts by a distance cut-off of $4.5 \AA$, and hydrogen bonds by the condition that the 
distance between the $\mathrm{N}$ and $\mathrm{O}$ atoms is less than $3.0 \AA$, and the angle of $\mathrm{N}-\mathrm{H} \cdots \mathrm{O}$ is between $160^{\circ}$ and $180^{\circ}$. Table 2 shows that presence of the inhibitors decreases the number of hydrogen bonds and hydrophobic contacts involved in packing of the two folds more than the ones stabilizing the stacking of chains. For example, in presence of R5S, we observe a $46 \%$ loss in contact at the packing interface, but the stacking contacts decrease by only $11 \%$. This loss of contacts at the packing interface is also reflected by an increase of about $29 \%$ in the inter-strand packing distance $\mathrm{D}_{\text {packing }}$ (shown also in Table 2) in presence of R5S. The corresponding change is much smaller for the other two inhibitors where $\mathrm{D}_{\text {packing }}$ increases by only about $2 \%$. Here, $\mathrm{D}_{\text {packing }}$ is defined by the distance between the center of mass of two chains at the interface where they are packed (residues 28-31).

\section{Mechanism of SAA fibril destabilization}

Our above analysis shows that R5S, the N-terminal sequence SAA protein (RSFFS), is more effective than the other two inhibitors in destabilizing the SAA fibril. Hence, we focus in the following on this inhibitor, trying to understand the mechanism by which R5S destabilizes SAA fibrils. For this purpose, we have first tried to quantify the destabilization of the SAA fibril by R5S by calculating the binding free energy of the chains. The later quantity is estimated by the MMPBSA approach ${ }^{35}$ as thermodynamic integration or other exact methods would have been too costly in terms of computation time, and values are listed in the Table $\mathbf{S 2}$ and Table $\mathbf{S 3}$ in the supporting information.

We find that presence of R5S reduces the fibril stability by about $60 \mathrm{Kcal} / \mathrm{mol}$, from $(-311 \pm 80)$ $\mathrm{Kcal} / \mathrm{mol}$ in the control to $(-254 \pm 60) \mathrm{Kcal} / \mathrm{mol}$. The loss of stability is mostly from the change in the packing free energy, i.e., the difference in free energy of the fibril $2 \mathrm{~F} 3 \mathrm{~L}$ and of two separated 
proto-fibrils 1F3L, which changes from $(-148 \pm 54) \mathrm{Kcal} / \mathrm{mol}$ for the control to $(-38 \pm 38) \mathrm{Kcal} / \mathrm{mol}$ in presence of the inhibitor. The changes in packing free energy of about $90 \mathrm{kcal} / \mathrm{mol}$ is due to a loss of interactions between residues of about $252 \mathrm{Kcal} / \mathrm{mol}$ that is only partially compensated by a gain in solvation free energy of about $-160 \mathrm{Kcal} / \mathrm{mol}$. A less pronounced difference is seen for the elongation free energy, i.e., the difference in free energy by adding one layer to the fibril (2F3L compared with $2 \mathrm{~F} 2 \mathrm{~L}+2 \mathrm{~F} 1 \mathrm{~L})$, which decreases by about $20 \mathrm{Kcal} / \mathrm{mol}$, from $(-143 \pm 54) \mathrm{Kcal} / \mathrm{mol}$ in the control to $(-125 \pm 53) \mathrm{Kcal} / \mathrm{mol}$ in presence of $\mathrm{R} 5 \mathrm{~S}$. The difference is again due to a reduction of interactions between residues, with the loss of about $56 \mathrm{Kcal} / \mathrm{mol}$ only partially compensated by a gain in the solvation free energy of about $-37 \mathrm{Kcal} / \mathrm{mol}$. Hence, our MMPBSA analysis indicates that the loss in fibril stability results from a loss of electrostatic and van der Waals interactions.

We have therefore compared in the next step the map of inter and intra residue distances measured in either the simulations where R5S inhibitor is present, or in the control (where neither R5S nor one of the other two inhibitors is present). These maps are shown in Figure 4, with the coloring marking the average distance between two residues. 

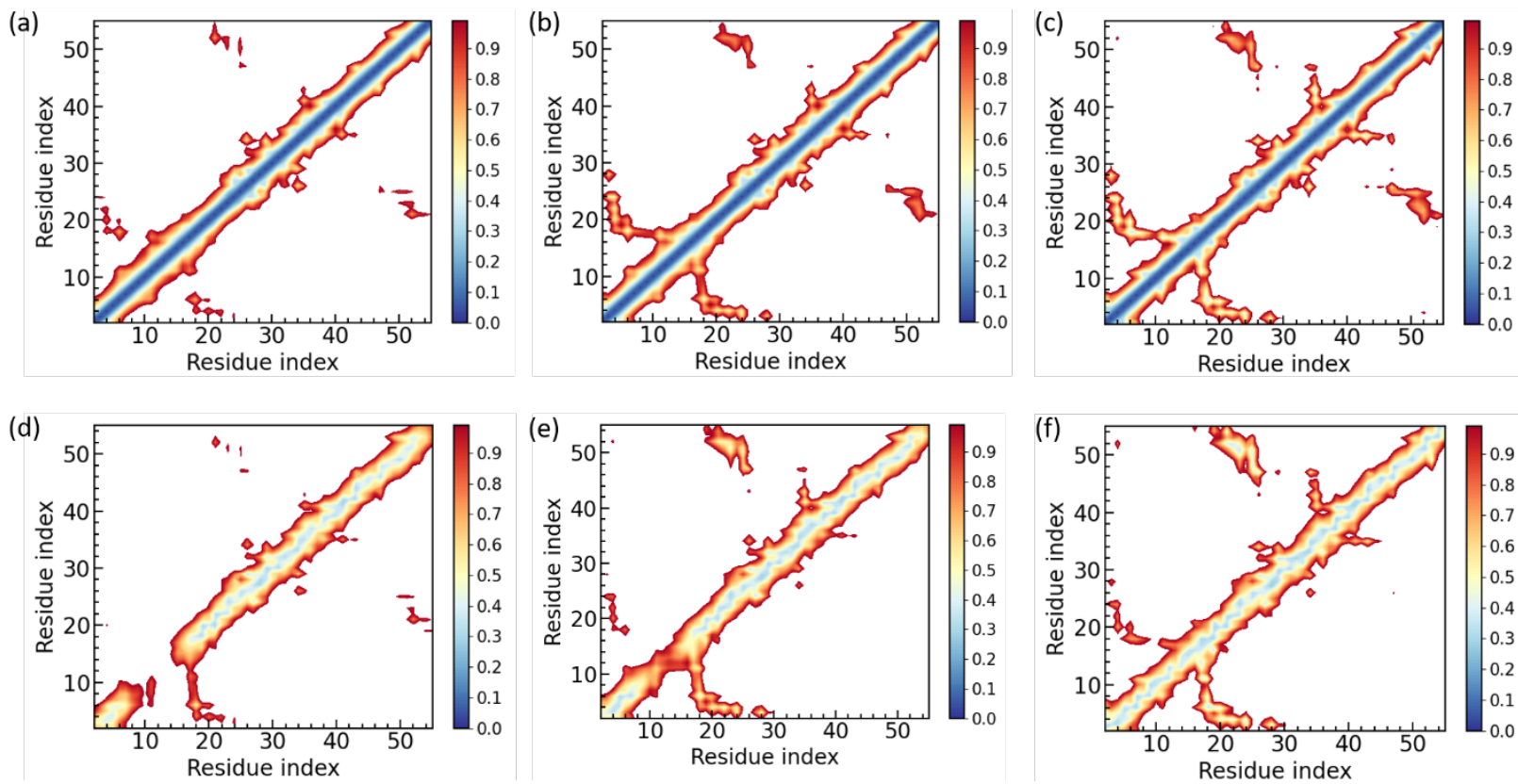

Figure 4: Intra-peptide side-chain distance map measured during simulations of SAA fibrils in the presence (a) and absence of (b) of peptide inhibitor R5S. The corresponding inter-peptide sidechain distance maps are also shown in (d) and (e), respectively. For a comparison, we also show (c) intrapeptide and (f) interpeptide side-chain distance maps of the cryo-EM SAA fibril model (PDB-ID: 6MST). Residue pairs whose average contact distance exceed $1.0 \mathrm{~nm}$ are excluded. Distance maps are calculated using the last $100 \mathrm{~ns}$ in all three independent trajectories.

Visual inspection of the cryo-EM structure (PDB-ID: 6MST) (resolved at $3.0 \AA$ resolution) together with the intra-chain and inter-chain distance map, derived from this structure and shown in Figure $4 \mathbf{c}$ and $\mathbf{4 f}$, demonstrate that the $\mathrm{N}$-terminal $\beta$-arch formed by residues $2-22$ is stabilized by the densely packed hydrophobic core formed by residues F4, F6, F11, M17, W18 and A20; and by salt bridges between residue-pairs D12-R15 and D16-R19. Of importance are also contacts between the C-terminal end encompassing residues 47-55 and the region encompassing residues 19-26 that stabilizes the C-terminal cavity, namely multiple hydrophobic contacts involving the residues A21, M24, W53, V52 and A54, and the salt bridges between E26-R47. These contacts are also seen in the control simulations, see Figure $\mathbf{4 b}$ and $\mathbf{4 e}$, indicating that these contacts are conserved in absence of any inhibitor. On the other hand, in presence of the peptide inhibitor R5S, are these contacts rarely found, see Figure 4a and 4d. Similarly, while only few contacts at the N- 
terminal Cross-stacking interface (encompassing residues 11-17) are lost in the control simulations (Figure 4e) the stacking contacts are lost in presence of R5S (Figure 4d), which may be one reason for its ability to destabilize the SAA fibrillar architecture as it causes a loss of van der Waals energy of about $56 \mathrm{Kcal} / \mathrm{mol}$ (see supplemental Tables S2 and S3).

Corresponding figures for the other two inhibitors, L4F and F5M, are shown in Figure S3 of the supporting information. Similar as in the case of R5S are the hydrophobic cluster at the N-terminus perturbed in presence of $\mathrm{L} 4 \mathrm{~F}$, as are the C-terminal cavity stabilizing contacts between the Cterminus (residues 47-55) and the residues 19-26. However, for F5M is the inter-stacking interface at the N-terminus conserved over the course of the simulation and the disruptive effect of F5M is even lower: the distance maps in Figure $\mathbf{S 3}$ do not show any obvious loss of contacts.

We next have calculated the residue-wise binding probability of R5S inhibitor towards the SAA fibril to understand the relation between R5S binding and the loss of intra-chain and inter-chain contacts. For this purpose, we define a binding site as the closest residue with at least one heavy atom within $4.5 \AA$ from the R5S and do not consider peptide inhibitors that move away from the fibril. Data are averaged over the final $100 \mathrm{~ns}$ of all three trajectories and shown in Figure 5. From the binding probability map we see that R5S preferentially binds with aromatic residues (F3, F4, F6, F11, W18 and Y21) hydrophobic residues (L7, A10, A14, M17 and A20), polar and charged residues (S2, S5, E9 and S22) at the N-terminus, disrupting the most amyloidogenic and hydrophobic segment of the protein. We also observe lower binding probability towards the packing interface through hydrophobic and polar interactions. As a result increases the interstrand packing distance by about $29 \%$. The resulting loss in contacts between polar and charged residues leads to a reduction of electrostatic energy between residues by about $219 \mathrm{Kcal} / \mathrm{mol}$, and 
of the van der Waals energy by about $34 \mathrm{Kcal} / \mathrm{mol}$, that decreases the packing energy of the fibril in presence of R5S (see supplemental Tables S2 and S3). Interestingly, we do not find any binding affinity towards the aromatic and hydrophobic residues at the C-terminal end (residues 53-55) and the GPGG motif (residues 48-51).

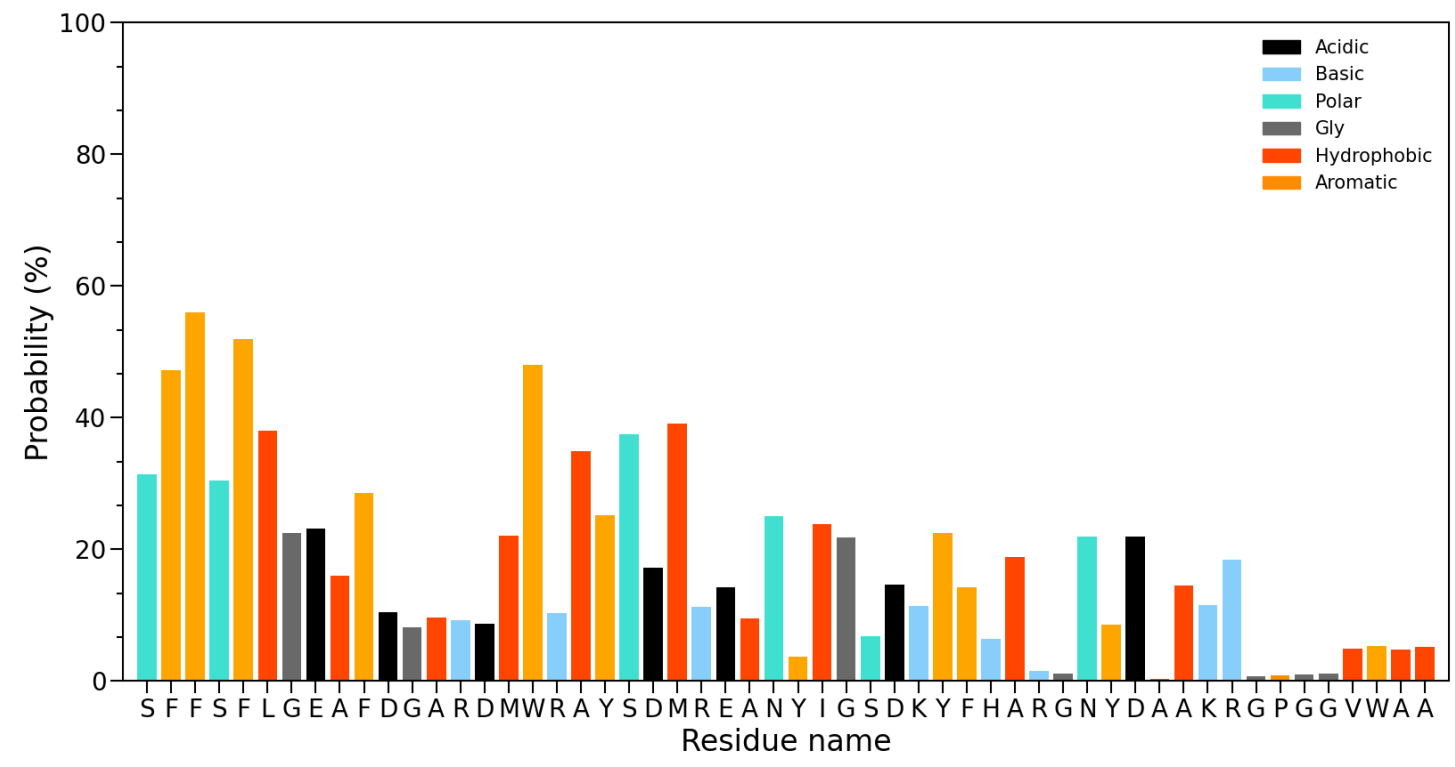

Figure 5: Residue-wise binding probability (normalized) of R5S inhibitor for the SAA fibril. Data are averaged over the final $100 \mathrm{~ns}$ of all three independent runs.

We remark that the binding affinities of L4F, shown in Figure $\mathbf{S 4}$ of the supporting information, is similar to R5S, but binds more towards certain aromatic and hydrophobic residues (e.g F3, F4 and M24), and less toward polar and charges residues. The differences reflect that L4F is purely hydrophobic while there are two polar serine $(\mathrm{S})$ and one positively-charged arginine $(\mathrm{R})$ residue in R5S inhibitor. Interestingly, while F5M (FVFLM) is also hydrophobic, we did not observe the same binding affinity to the hydrophobic and amyloidogenic N-terminus, see Figure S5 in the supporting information, as we observed for either R5S or L4F. 
As discussed above, our simulations show that all three small peptides interact with the SAA fibril mostly by hydrophobic interactions and $\pi-\pi$ stacking. In the case of R5S there is additional stabilization by long-range electrostatic interactions involving the polar (S) and charged (R) residues of R5S. These additional, interactions lead to the strong binding of all six R5S inhibitors to the SAA fibril fragment (a hexamer). On the other hand, in the case of L4F and F5M are fewer of the peptides bound to the fibril. For example, in one of the L4F inhibitor trajectory, we find after $250 \mathrm{~ns}$ only two peptides still binding to the fibril. Hence, our data demonstrate the need to consider polar and charged residues in the design of peptides inhibiting SAA amyloidosis as sole hydrophobic interactions are not sufficient for a strong binding.

\section{Destabilization of SAA fibrils by R5S inhibitor as function of fibril size}

Our above study established that the peptide inhibitor R5S, SAA $1-5$ (RSFFS), is able to destabilize small fibril fragments such as the two-fold three-layer $2 \mathrm{~F} 3 \mathrm{~L}$. While the hexamer is above the critical size for SAA fibril stability ${ }^{25}$, a potential application as a drug would require that R5S inhibitor can also dissolve fully-grown SAA fibrils. Hence, in order to test whether the destabilizing effect of R5S persist with increasing number of layers, we have also simulated the 2F4L octamer as the next larger SAA assembly. Comparing the time evolution of the global RMSD in Figure 6, measured in trajectories derived with either R5S inhibitor present or absent, we still observe the destabilizing effect of R5S seen in simulations of the smaller 2F3L hexamer. While the stability of the SAA fibril increases with size, and therefore the measured RMSD values are smaller and increasing slower, the qualitative behavior is the same as seen in the simulations of the smaller $2 \mathrm{~F} 3 \mathrm{~L}$ fibril. While the $2 \mathrm{~F} 4 \mathrm{~L}$ octamer is still a small system, we believe that this 
similarity to the $2 \mathrm{~F} 3 \mathrm{~L}$ hexamer indicates that the destabilizing effect of R5S inhibitor will likely persist for the much larger systems one would observe in vito or in vivo.

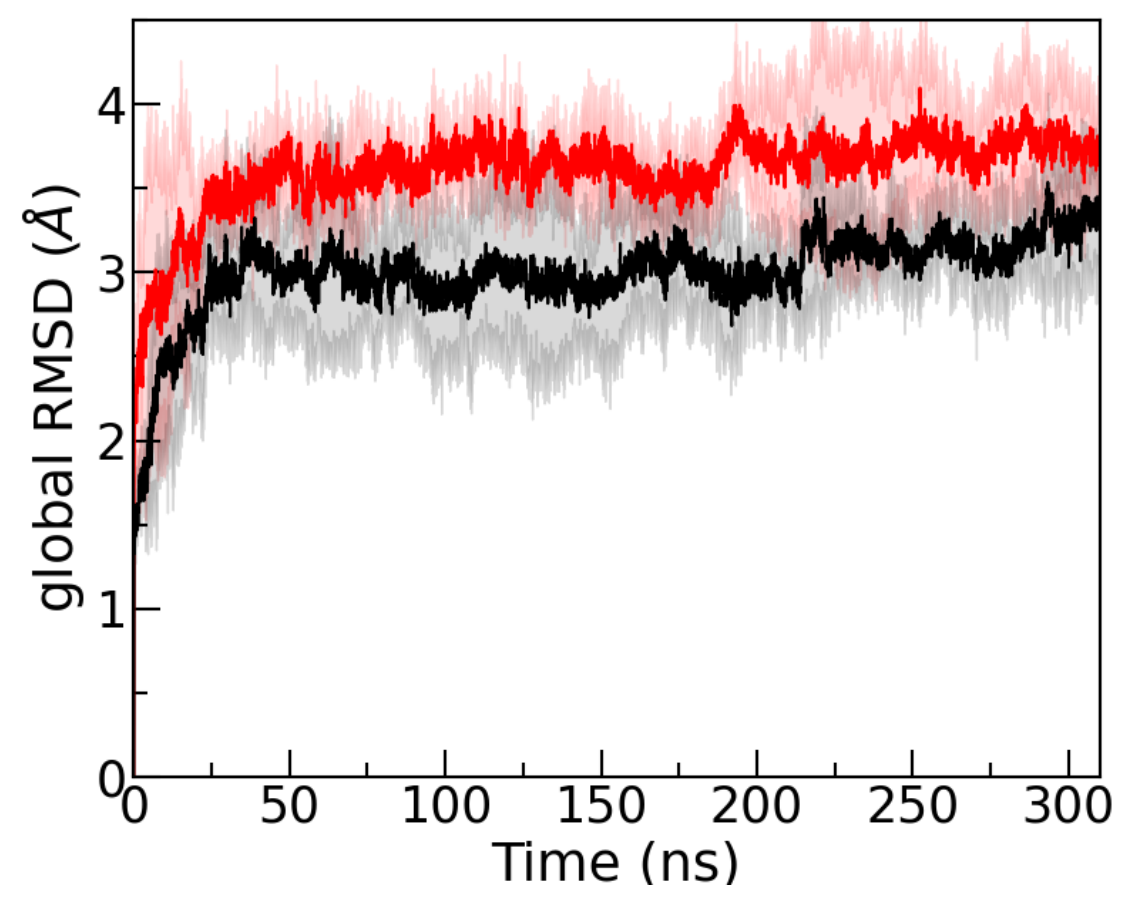

Figure 6: Time evolution of the global RMSD measured in simulations of the two-fold-four-layer (2F4L) SAA fibril in absence (in black) and presence of R5S inhibitor (in red). The shaded region represents the standard deviation. The RMSD values are calculated in reference to the experimentally resolved structure considering all backbone atoms.

\section{Destabilization of SAA fibrils by D-R5S and DRI-R5S}

In the preceding section we have shown that the peptide inhibitor R5S destabilizes SAA fibrils. This destabilization is a minimum condition for use of R5S as a potential drug candidate inhibiting SAA amyloid formation. However, R5S can only act as a drug if it survives long enough in the body to be able to dissolve SAA aggregates. However, peptide-based therapies are often hampered by short half-life times, a likely complication for the use of R5S. For this reason, we have also studied versions of R5S where the L-amino acids are replaced by D-amino acids, as such peptides are resistant to proteolytic digestion resulting in longer half-life times. Hence, following our earlier 
work ${ }^{21}$ we have studied both a variant where the sequence is unchanged (D-R5S), and one where also the sequence is reversed (DRI-R5S). The later variant is chosen because such D-retro-inverso peptides resemble the parent peptide and often have similar biological activity. ${ }^{22-24}$

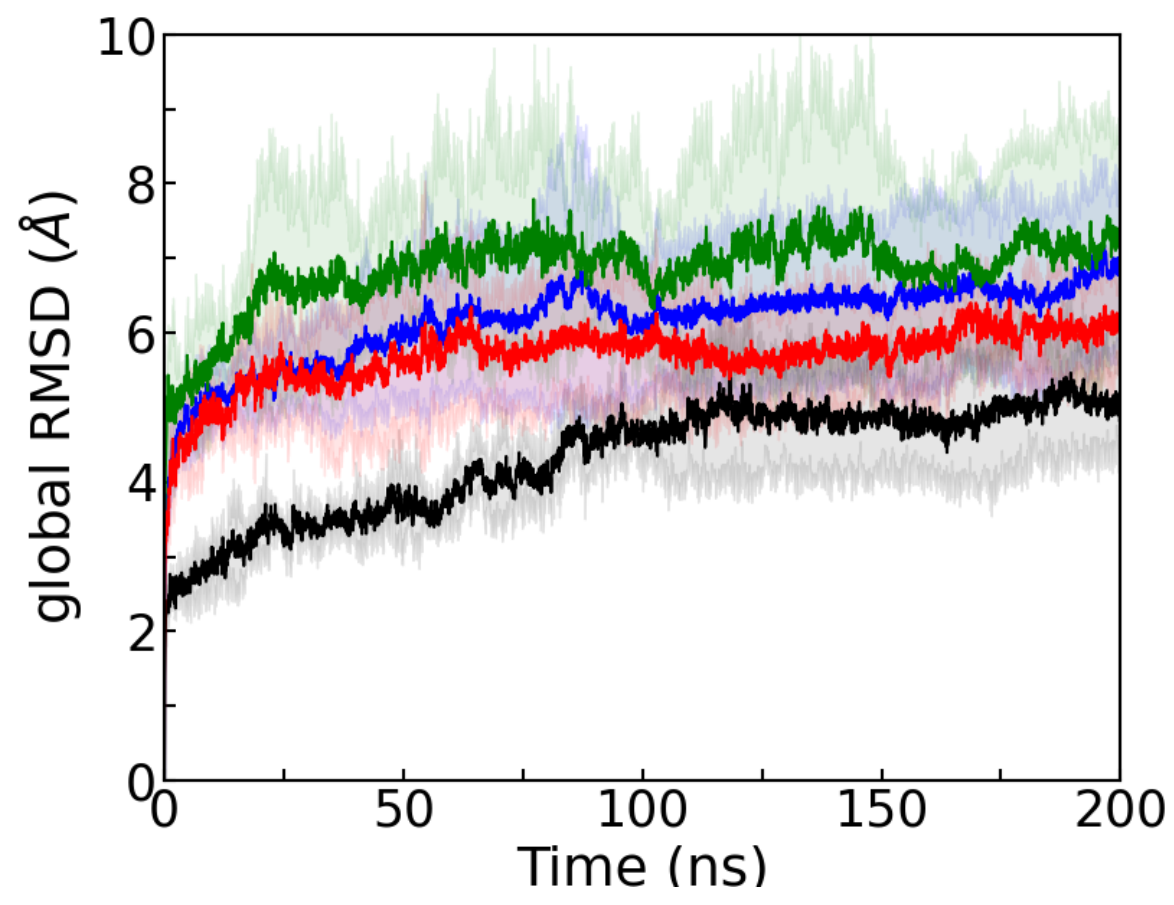

Figure 7: Time evolution of the global RMSD measured in simulations of the two-fold-three-layer (2F3L) SAA fibril in presence of DRI-R5S (blue) and D-R5S (red). For a comparison, we show also corresponding data obtained from simulations in presence of L-R5S (green), and from the control simulation (black). The shaded region represents the standard deviation. The RMSD values are calculated in reference to the experimentally resolved structure considering all backbone atoms.

Our results are shown in Figure 7, where we draw the time evolution of the global RMSD of the SAA fibril relative to the initial cryo-EM structure. Shown are the results from simulations in presence of DRI-R5S or D-R5S and the corresponding data obtained from simulations without any inhibitor or with L-R5S present. RMSD data over the course of three separate $200 \mathrm{~ns}$ 
simulations for each system indicate that the DRI-R5S is more effective to destabilize the fibril compared to D-R5S: the averaged RMSD values over the final $100 \mathrm{~ns}$ and three trajectories are $(0.75 \pm 0.04) \mathrm{nm}$ and $(0.62 \pm 0.02) \mathrm{nm}$ in presence of DRI-R5S and D-R5S, respectively.

Table 3: Mean values of solvent accessible surface area (SASA), number of stacking contacts $\left(\mathrm{N}_{\text {stacking }}\right)$; and of the number of packing contacts $\left(\mathrm{N}_{\text {packing }}\right)$; and of the inter-strand packing distance $\left(D_{\text {packing}}\right)$. Averages are calculated over the three independent trajectories considering only the final $100 \mathrm{~ns}$ of each trajectory. The corresponding standard deviations are listed in brackets. We also provide percent changes to describe the change of values calculated from simulations in presence of an inhibitor, compared to the values calculated in the control simulations.

\begin{tabular}{|c|c|c|c|c|}
\hline System & DRI-R5S & D-R5S & Control & L-R5S \\
\hline SASA $\left(\AA^{2}\right)$ & $7712 \pm 574$ & $7174 \pm 369$ & $6435 \pm 371$ & $8118 \pm 632$ \\
\hline$\% \Delta$ SASA & 20 & 11 & - & 26 \\
\hline $\mathrm{N}_{\text {stacking }}$ & $14420 \pm 498$ & $15452 \pm 679$ & $16100 \pm 376$ & $14389 \pm 612$ \\
\hline$\% \Delta \mathrm{N}_{\text {stacking }}$ & -10 & -4 & - & -11 \\
\hline $\mathrm{N}_{\text {packing }}$ & $1529 \pm 124$ & $1626 \pm 252$ & $2265 \pm 216$ & $1203 \pm 425$ \\
\hline$\% \Delta \mathrm{N}_{\text {packing }}$ & -32 & -28 & - & -47 \\
\hline $\mathrm{D}_{\text {packing }}(\AA)$ & $9.5 \pm 0.3$ & $9.6 \pm 0.4$ & $9.5 \pm 0.3$ & $12.2 \pm 4.0$ \\
\hline
\end{tabular}

This observation is corroborated by the values of the hydrophobic SASA, $\mathrm{N}_{\text {stacking }}, \mathrm{N}_{\mathrm{HB} \text {-stacking, }}$

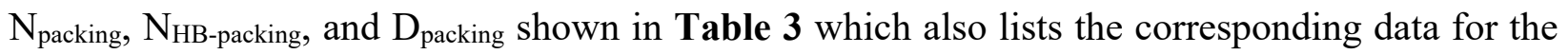
control and the L-amino acid version of R5S. For example, the hydrophobic SASA increases by $\sim 21.6 \%$ in presence of DRI-R5S, while corresponding change in presence of D-R5S is about $12.7 \%$. Percentage change in most of these quantities in relation to the control are comparable for DRI-R5S with the corresponding changes for L-R5S, see Table 3. While we do not find in presence of either DRI-R5S or D-R5S the increase in inter-strand packing distance that we see for L-R5S, taken together our data do suggest that DRI-R5S has a similar effectiveness than L-R5S in destabilizing SAA fibrils. The inter and intra chain distance maps in presence of DRI-R5S that we show in Figure $\mathbf{S 6}$ of the supporting information add further evidence for this conclusion. The distance map is similar to that of L-R5S, with hydrophobic cluster, salt-bridges and the N-terminal 
inter-stacking interfaces disrupted in presence of DRI-R5S. The binding probability map in Figure S7 also shows similar binding models as L-R5S since DRI-R5S is again strongly binding to hydrophobic and aromatic residues in the N-terminal hydrophobic cluster or forming salt bridges with the charged residues E9, D12, D33 and K34.

\section{Conclusions}

SAA Amyloidosis is a common secondary disease connect with various cancer and inflammatory diseases $^{1,2}$, and may also be a risk after SARS-COV-2 infections ${ }^{8}$. In this paper we have studied three peptides that may serve as drug candidates for inhibiting or slowing down SAA amyloid formation. Using extensive all-atom molecular dynamics simulations, we compare the ability of the N-terminal sequence $\mathrm{SAA}_{1-5}(\mathrm{R} 5 \mathrm{~S})$, the $\mathrm{A} \beta$ peptide segment $\mathrm{A} \beta_{17-20}(\mathrm{~L} 4 \mathrm{~F})$, and the peptide FVFLM (F5M) of the protein SERPINA, to destabilize a small fragment of the experimentally resolved human SAA fibril (PDB-ID: 6MST). We find that the de-stabilizing effect is larger for the amphipathic R5S inhibitor than for the purely hydrophobic L4F and F5M, and persist when increasing the fragment size. Going beyond confirming previous experimental results ${ }^{10}$, our numerical investigation allows us to propose a mechanism for the inhibitor properties of R5S. We find that this peptide binds to the N-terminus, the most amyloidogenic and hydrophobic segment of the SAA fibril, mainly through short-range hydrophobic and $\pi-\pi$ stacking interactions with aromatic and hydrophobic residues, but unlike for L4F and F5M inhibitors is the binding interface in addition stabilized via long-range interactions involving the polar and charged residues of R5S. The complex binding patterns of hydrophobic, polar and ionic interactions disturbs not only the densely packed hydrophobic core at N-terminus but also the network of contacts that stabilize the 
SAA fibril geometry. From these observations we conclude that for the design of peptide inhibitors against SAA fibril formation, one should optimize not only hydrophobic contacts of the peptide to the N-terminus, but also salt bridge formation and polar contacts with the charged residues in this segment. In our case it is the presence of such contacts what set R5S inhibitor apart from the other two peptides where the binding to the N-terminus is solely by hydrophobic contacts. Our proposed mechanism is consistent with the crucial role of the $\mathrm{N}$-terminus (the first eleven residues) as start point for the SAA fibril self-assembly ${ }^{37}$. Hence, it is plausible that drug candidates such as our R5S inhibitor will have to target this domain, both to inhibit binding of chains in the early stages of the amyloidogenic pathway, and to destabilize the aggregates.

We finally have addressed a common problem in the use of peptides such as R5S as drug candidates: short half-life time and the resulting need for frequent administration that makes their use cumbersome. Following common protocols we have therefore also studied versions of R5S where the L-amino-acids are replaced by their mirror D-amino acids, leading to peptides that are resistant to proteolytic digestion and have longer half-life times. Especially, we find that DRI-R5S, where not only L-amino acids are replaced by D-amino acids but also the sequence reversed, has similar inhibitory effects than the original L-R5S. The much longer life-time may make DRI-R5S an attractive drug candidate targeting SAA amyloidosis. We hope that the inhibitory potency of DRI-R5S can be tested soon in suitable experiments.

\section{Acknowledgments}


The simulations in this work were done using the SCHOONER cluster of the University of Oklahoma, XSEDE resources allocated under grant MCB160005 (National Science Foundation), and TACC resources allocated under grant under grant MCB20016 (National Science Foundation). We acknowledge financial support from the National Institutes of Health under grant GM120634. We would like to thank Alan J. Ray for providing guidance and scripts for making figures.

\section{References}

(1) Caughey, B.; Baron, G. S. Are Cheetahs on the Run from Prion-like Amyloidosis? Proc. Natl. Acad. Sci. 2008, 105 (20), 7113 LP - 7114.

(2) Papendick, R. E.; Munson, L.; O’Brien, T. D.; Johnson, K. H. Systemic AA Amyloidosis in Captive Cheetahs (Acinonyx Jubatus). Vet. Pathol. 1997, 34 (6), 549-556.

(3) Gillmore, J. D.; Lovat, L. B.; Persey, M. R.; Pepys, M. B.; Hawkins, P. N. Amyloid Load and Clinical Outcome in AA Amyloidosis in Relation to Circulating Concentration of Serum Amyloid A Protein. The Lancet 2001, 358 (9275), 24-29.

(4) Kofman, T.; Grimbert, P.; Canouï-Poitrine, F.; Zuber, J.; Garrigue, V.; Mousson, C.; Frimat, L.; Kamar, N.; Couvrat, G.; Bouvier, N.; Albano, L.; Le Thuaut, A.; Pillebout, E.; Choukroun, G.; Couzi, L.; Peltier, J.; Mariat, C.; Delahousse, M.; Buchler, M.; Le Pogamp, P.; Bridoux, F.; Pouteil-Noble, C.; Lang, P.; Audard, V. Renal Transplantation in Patients With AA Amyloidosis Nephropathy: Results From a French Multicenter Study. Am. J. Transplant. 2011, 11 (11), 2423-2431.

(5) Cheng, L.; Yang, J.-Z.; Bai, W.-H.; Li, Z.-Y.; Sun, L.-F.; Yan, J.-J.; Zhou, C.-L.; Tang, B.P. Prognostic Value of Serum Amyloid A in Patients with COVID-19. Infection 2020, 48 (5), 715-722. 
(6) Li, H.; Xiang, X.; Ren, H.; Xu, L.; Zhao, L.; Chen, X.; Long, H.; Wang, Q.; Wu, Q. Serum Amyloid A Is a Biomarker of Severe Coronavirus Disease and Poor Prognosis. J. Infect. 2020, $80(6), 646-655$.

(7) Hanff, T. C.; Mohareb, A. M.; Giri, J.; Cohen, J. B.; Chirinos, J. A. Thrombosis in COVID19. Am. J. Hematol. 2020, 95 (12), 1578-1589.

(8) Jana, A. K.; Greenwood, A. B.; Hansmann, U. H. E. Presence of a SARS-CoV-2 Protein Enhances Amyloid Formation of Serum Amyloid A. J. Phys. Chem. B 2021, 125 (32), 9155-9167.

(9) Scrocchi, L. A.; Chen, Y.; Waschuk, S.; Wang, F.; Cheung, S.; Darabie, A. A.; McLaurin, J.; Fraser, P. E. Design of Peptide-Based Inhibitors of Human Islet Amyloid Polypeptide Fibrillogenesis. J. Mol. Biol. 2002, 318 (3), 697-706.

(10) Sosnowska, M.; Skibiszewska, S.; Kamińska, E.; Wieczerzak, E.; Jankowska, E. Designing Peptidic Inhibitors of Serum Amyloid A Aggregation Process. Amino Acids 2016, 48 (4), 1069-1078.

(11) El-Agnaf, O. M. A.; Paleologou, K. E.; Greer, B.; Abogrein, A. M.; King, J. E.; Salem, S. A.; Fullwood, N. J.; Benson, F. E.; Hewitt, R.; Ford, K. J.; Martin, F. L.; Harriott, P.; Cookson, M. R.; Allsop, D. A Strategy for Designing Inhibitors of $\alpha$-Synuclein Aggregation and Toxicity as a Novel Treatment for Parkinson's Disease and Related Disorders. FASEB J. 2004, 18 (11), 1315-1317.

(12) Skibiszewska, S.; Żaczek, S.; Dybala-Defratyka, A.; Jędrzejewska, K.; Jankowska, E. Influence of Short Peptides with Aromatic Amino Acid Residues on Aggregation Properties of Serum Amyloid A and Its Fragments. Arch. Biochem. Biophys. 2020, 681, 108264.

(13) Tjernberg, L. O.; Näslund, J.; Lindqvist, F.; Johansson, J.; Karlström, A. R.; Thyberg, J.; 
Terenius, L.; Nordstedt, C. Arrest of -Amyloid Fibril Formation by a Pentapeptide Ligand (*). J. Biol. Chem. 1996, 271 (15), 8545-8548.

(14) Soto, C.; Sigurdsson, E. M.; Morelli, L.; Asok Kumar, R.; Castaño, E. M.; Frangione, B. $\beta$ Sheet Breaker Peptides Inhibit Fibrillogenesis in a Rat Brain Model of Amyloidosis: Implications for Alzheimer's Therapy. Nat. Med. 1998, 4 (7), 822-826.

(15) Sato, T.; Kienlen-Campard, P.; Ahmed, M.; Liu, W.; Li, H.; Elliott, J. I.; Aimoto, S.; Constantinescu, S. N.; Octave, J.-N.; Smith, S. O. Inhibitors of Amyloid Toxicity Based on $\beta$-Sheet Packing of A $\beta 40$ and A $\beta 42$. Biochemistry 2006, 45 (17), 5503-5516.

(16) Wang, W.; Xi, W.; Hansmann, U. H. E. Stability of the N-Terminal Helix and Its Role in Amyloid Formation of Serum Amyloid A. ACS Omega 2018, 3 (11), 16184-16190.

(17) Egashira, M.; Takase, H.; Yamamoto, I.; Tanaka, M.; Saito, H. Identification of Regions Responsible for Heparin-Induced Amyloidogenesis of Human Serum Amyloid A Using Its Fragment Peptides. Arch. Biochem. Biophys. 2011, 511 (1), 101-106.

(18) Buhimschi, I. A.; Nayeri, U. A.; Zhao, G.; Shook, L. L.; Pensalfini, A.; Funai, E. F.; Bernstein, I. M.; Glabe, C. G.; Buhimschi, C. S. Protein Misfolding, Congophilia, Oligomerization, and Defective Amyloid Processing in Preeclampsia. Sci. Transl. Med. 2014, 6 (245), 245ra92 LP-245ra92.

(19) Kouza, M.; Banerji, A.; Kolinski, A.; Buhimschi, I. A.; Kloczkowski, A. Oligomerization of FVFLM Peptides and Their Ability to Inhibit Beta Amyloid Peptides Aggregation: Consideration as a Possible Model. Phys. Chem. Chem. Phys. 2017, 19 (4), 2990-2999.

(20) Liberta, F.; Loerch, S.; Rennegarbe, M.; Schierhorn, A.; Westermark, P.; Westermark, G. T.; Hazenberg, B. P. C.; Grigorieff, N.; Fändrich, M.; Schmidt, M. Cryo-EM Fibril Structures from Systemic AA Amyloidosis Reveal the Species Complementarity of 
Pathological Amyloids. Nat. Commun. 2019, 10 (1), 1104.

(21) Pandey, P.; Nguyen, N.; Hansmann, U. H. E. D-Retro Inverso Amylin and the Stability of Amylin Fibrils. J. Chem. Theory Comput. 2020, 16 (8), 5358-5368.

(22) Koutsopoulos, S. Peptide Applications in Biomedicine, Biotechnology and Bioengineering; Woodhead Publishing, 2017.

(23) Feng, Z.; Xu, B. Inspiration from the Mirror: D-Amino Acid Containing Peptides in Biomedical Approaches. Biomol. Concepts 2016, 7 (3), 179-187.

(24) Biswas, N.; Gayen, J.; Mahata, M.; Su, Y.; Mahata, S. K.; O’Connor, D. T. Novel Peptide Isomer Strategy for Stable Inhibition of Catecholamine Release: Application to Hypertension. Hypertension (Dallas, Tex. : 1979) 2012, 60 (6), 1552-1559.

(25) Wang, W.; Hansmann, U. H. E. Stability of Human Serum Amyloid A Fibrils. J. Phys. Chem. B 2020, 124 (47), 10708-10717.

(26) Pettersen, E. F.; Goddard, T. D.; Huang, C. C.; Couch, G. S.; Greenblatt, D. M.; Meng, E. C.; Ferrin, T. E. UCSF Chimera-A Visualization System for Exploratory Research and Analysis. J. Comput. Chem. 2004, 25 (13), 1605-1612.

(27) Trott, O.; Olson, A. J. AutoDock Vina: Improving the Speed and Accuracy of Docking with a New Scoring Function, Efficient Optimization, and Multithreading. J. Comput. Chem. 2010, $31(2), 455-461$.

(28) Jorgensen, W. L.; Chandrasekhar, J.; Madura, J. D.; Impey, R. W.; Klein, M. L. Comparison of Simple Potential Functions for Simulating Liquid Water. J. Chem. Phys. 1983, 79 (2), 926-935.

(29) Bussi, G.; Donadio, D.; Parrinello, M. Canonical Sampling through Velocity Rescaling. $J$. Chem. Phys. 2007, 126 (1), 14101. 
(30) Parrinello, M.; Rahman, A. Polymorphic Transitions in Single Crystals: A New Molecular Dynamics Method. J. Appl. Phys. 1981, 52 (12), 7182-7190.

(31) Hess, B.; Bekker, H.; Berendsen, H. J. C.; Fraaije, J. G. E. M. LINCS: A Linear Constraint Solver for Molecular Simulations. J. Comput. Chem. 1997, 18 (12), 1463-1472.

(32) Miyamoto, S.; Kollman, P. A. Settle: An Analytical Version of the SHAKE and RATTLE Algorithm for Rigid Water Models. J. Comput. Chem. 1992, 13 (8), 952-962.

(33) Essmann, U.; Perera, L.; Berkowitz, M. L.; Darden, T.; Lee, H.; Pedersen, L. G. A Smooth Particle Mesh Ewald Method. J. Chem. Phys. 1995, 103 (19), 8577-8593.

(34) Humphrey, W.; Dalke, A.; Schulten, K. VMD: Visual Molecular Dynamics. J. Mol. Graph. 1996, $14(1), 33-38$.

(35) Luo, R.; David, L.; Gilson, M. K. Accelerated Poisson-Boltzmann Calculations for Static and Dynamic Systems. J. Comput. Chem. 2002, 23 (13), 1244-1253.

(36) Kumari, R.; Kumar, R.; Lynn, A. G_mmpbsa-A GROMACS Tool for High-Throughput MM-PBSA Calculations. J. Chem. Inf. Model. 2014, 54 (7), 1951-1962.

(37) Wang, W.; Khatua, P.; Hansmann, U. H. E. Cleavage, Downregulation, and Aggregation of Serum Amyloid A. J. Phys. Chem. B 2020, 124 (6), 1009-1019. 